\title{
Tactile-based Compliance with Hierarchical Force Propagation for Omnidirectional Mobile Manipulators
}

\author{
Quentin Leboutet, Emmanuel Dean-León and Gordon Cheng \\ Institute for Cognitive Systems (ICS) \\ Technical University of Munich, Germany \\ Contact: \{quentin. leboutet, dean, gordon\}@tum.de
}

\begin{abstract}
In this paper, we address the issue of wholebody compliance with hierarchical force propagation for omnidirectional mobile manipulators. Using the multimodal tactile feedback (force and proximity) provided by an artificial skin, our objective is to generate prioritized compliant behaviors in response to multicontact physical interactions between a mobile robot and its external environment. To be more specific the force components which cannot be properly propagated using upper-limbs must be projected onto the mobile base in order to generate suitable compliant motions. Using Quadratic Programming (QP), we propose a general method allowing to properly estimate and propagate these force components while respecting the robot physical constraints. In this way, robust whole-body compliance can be achieved. We finally test and validate our method on a complex robotic system.
\end{abstract}

Index Terms-Virtual compliance, Quadratic programming, Hierarchical control, Force propagation, Multimodal robot skin.

\section{INTRODUCTION}

\section{A. Motivation}

Nowadays, mobile robots are expected to perform a wide variety of demanding tasks while remaining perfectly safe for humans, with whom they - intentionally or accidentally - interact. In this context, compliance is of primary importance since it allows robots to limit, if not to control the external forces applied to their body, by generating suitable reactive motions. Greatly reducing the risk of both injury and mechanical damages, compliance was widely investigated over the past two decades [1], [2], [4]. Nevertheless, its implementation on mobile robots remains difficult since it requires a proper coordination between their upper and lower body. When Humans interact with their environment, they coordinate their upper and lower limbs in a very fluid and elegant way: naturally compliant, the arm movements are smoothly complemented by those of the legs as soon as their posture becomes "uncomfortable" due to kinematic or dynamic constraints. Transposed to the case of wheeled mobile manipulators, this assumes the existence of an underlying hierarchy between the arms and the mobile base, prioritizing the use of the first ones to propagate the external forces and projecting the unfeasible - or "hardly feasible" - constraints onto the second one to maintain compliance (Fig. 1).

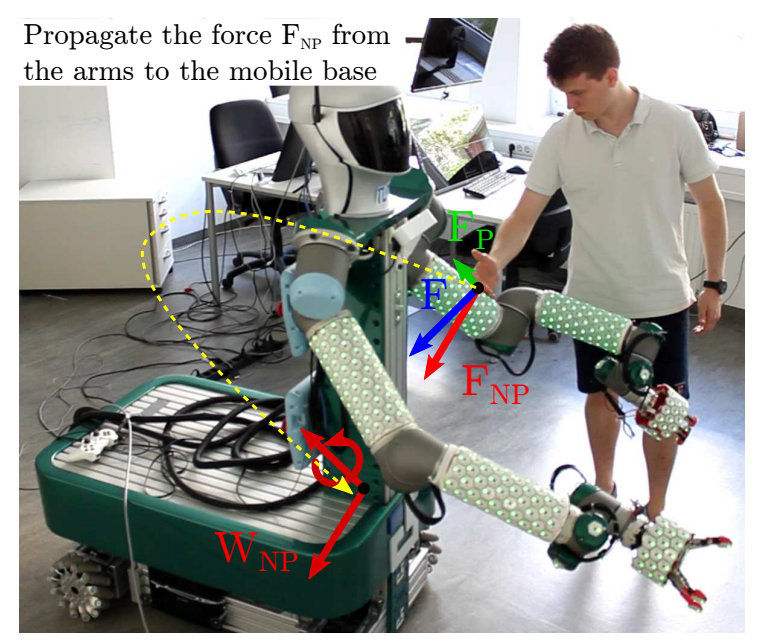

Fig. 1. Our experimental platform, TOMM (Tactile Omnidirectional Mobile Manipulator), is covered with a multimodal artificial skin. The forces applied to the robot are sensed by the skin and propagated along the upper limbs. Only the improperly propagated force components - in red on the figureare projected onto the mobile base in order to generate suitable compliant motions, when the arms can no longer move in a given direction of space.

\section{B. Related Work}

Little research have been directly dealing with wholebody compliance for wheeled mobile manipulators. In fact, most of the presented works only consider one aspect of the problem, namely upper or lower limb compliance but rarely both at the same time. For instance in [5], [6], a set of force and position sensors placed in some key locations of a compliant upper body, provide direct control over the mobile base movements, but upper and lower limbs compliance problems are treated separately. In [7], [8], the issue of upperbody control with active compliant torso is treated using an impedance-admittance control strategy. This approach is interesting since it allows the external constraints applied to the robot, to be treated on the same level as any other control objective as long as they can be detected by suitable sensor modalities. Compliance can therefore be achieved while formally respecting some of the robot's kinematic and dynamic constraints (such as self-collision or joint limits avoidance) [9] or can even be included in a stack of task architecture [10]. However, since it is not possible in the case of joint 
torque feedback, to detect nullspace components of external forces applied to the arms kinematic chain, an additional force/torque sensor modality is required on the mobile base [11]-[13] in order to achieve whole-body compliance.

\section{Our Approach}

Realizing compliant behaviors on an industrial manipulator is often difficult since major structural modifications are necessary to provide the robot with proper sensor modalities. In this context, the use of external force sensors is interesting but still highly limited by either spatial resolution or by cost. The concept of tactile feedback, namely the possibility of covering a robot with an artificial skin in order to get suitable force feedback without major structural changes was thus investigated over the last decade and several prototypes were developed [14], [15]. The artificial skin developed in our institute (Fig. 2.b) is the first of its kind since it is multimodal - namely capable of measuring contact forces, proximity, temperature, vibrations and even orientation thereby providing robots with an unprecedented awareness of their close-contact environment (Fig. 2.b and Fig. 2.c). Being flexible, versatile and low-cost, this skin can be adapted on a wide range of manipulators and used for active compliance realization. Thenceforth, the main focus of this work is to demonstrate that it is possible to realize active whole-body compliance on a non-compliant multi-limb industrial mobile manipulator, based on the force and proximity feedback provided by such an artificial skin. This paper is organized as follows: the Section II provides a formal description of the hierarchical force propagation problem. The Section III then describes the proposed approach to perform hierarchical force propagation using tactile feedback and quadratic programming. The experiments and results are presented and discussed in Section IV. Finally, Section V gives a short conclusion.

\section{Problem Formulation}

The general hierarchical compliance problem can be formulated as follows: we want to find the torque $\tau_{\text {ext }}$ to be applied on each actuator, such that the external forces acting on the robot can produce safe reactive motions while respecting both its physical constraints and limb hierarchy. Disregarding the issues of tilting or instability, we describe our holonomic mobile base as a set of two orthogonal prismatic joints and one revolute joint, respectively along the $x, y$ and $z$ axes of Cartesian space. These 3 extra Degrees of Freedom (DOF) are added to the ones of upper-limb's kinematic chains. The coupling between upper-limbs is here neglected. The general expression of the equations of motion for such a robot can be obtained using Euler-Lagrange's formalism [16]:

$$
\mathbf{M}(\mathbf{q}) \ddot{\mathbf{q}}+\mathbf{C}(\mathbf{q}, \dot{\mathbf{q}}) \dot{\mathbf{q}}+\mathbf{G}(\mathbf{q})+\beta \dot{\mathbf{q}}=\tau_{c t r l}+\tau_{e x t}
$$

where $\mathbf{q}=\left[\theta^{\top}, \mathbf{x}_{b}^{\top}\right]^{\top}$ is the generalized position vector (depicting upper limbs $\theta \in \mathbb{R}^{n}$ and mobile base positions $\mathbf{x}_{b} \in \mathbb{R}^{3}$ respectively), $\mathbf{M}(\mathbf{q}) \in \mathbb{R}^{(n+3) \times(n+3)}$ is the

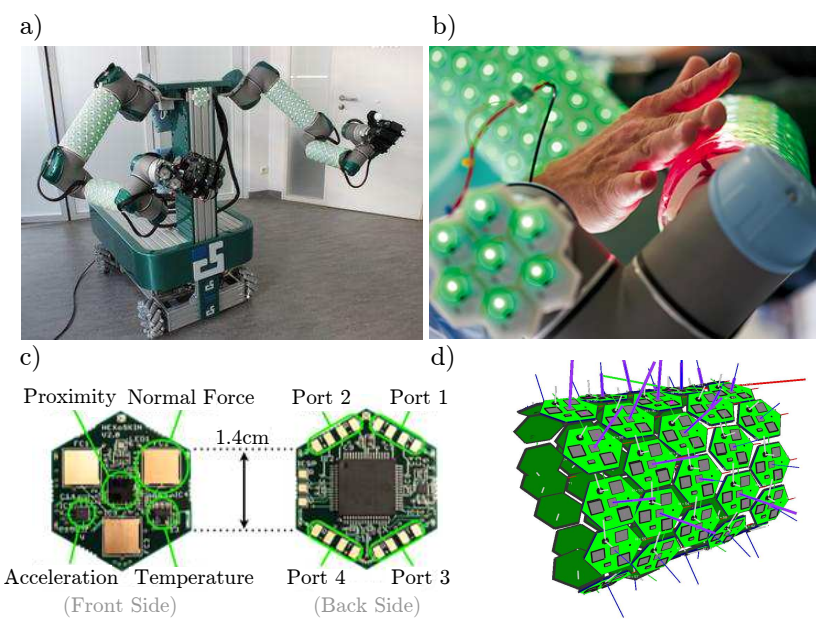

Fig. 2. a): Our robot TOMM. b): TOMM's artificial skin reacts to both proximity and to external forces, fusing these informations as a set of cell-specific force vectors $\mathbf{F}_{\text {cell }_{i}}$, given in each arm's base frame. c): The artificial skin mounted on TOMM was developed in our research institute at the Technical University of Munich. It is a redundant network of independent cells organized in patches. Each skin cell has a set of three normal force sensors, a temperature sensor, a proximity sensor and an accelerometer [15]. d): Shape recognition using the artificial skin.

Generalized Inertia matrix, $\mathbf{C}(\mathbf{q}, \dot{\mathbf{q}}) \in \mathbb{R}^{(n+3) \times(n+3)}$ is the Coriolis and Centripetal effects matrix, $\mathbf{G}(\mathbf{q}) \in \mathbb{R}^{n+3}$ is the Gravitational torque vector, $\beta \in \mathbb{R}^{(n+3) \times(n+3)}$ is a Viscous friction operator, $\tau_{c t r l} \in \mathbb{R}^{n+3}$ is the vector of control torques and $\tau_{\text {ext }} \in \mathbb{R}^{n+3}$ depicts the torque produced on each joint by the external forces. Usually $\tau_{\text {ext }}$ is formulated as the additive contributions of each external forces, propagated along the robot kinematic chain:

$$
\tau_{\text {ext }}=\sum_{i=1}^{k} \mathbf{J}_{i}^{\top}(\mathbf{q}) \mathbf{F}_{e x t_{i}}
$$

where each of the $k$ external constraints $\mathbf{F}_{\text {ext }_{i}} \in \mathbb{R}^{m}$ applied to the robot, is provided with a specific Jacobian matrix $\mathbf{J}_{i}(\mathbf{q})=\frac{\partial \mathbf{x}_{i}}{\partial \mathbf{q}} \in \mathbb{R}^{m \times(n+3)}$. Since we want to perform hierarchical force propagation between upper and lower limbs, both the external force vector $\mathbf{F}_{\text {ext }}$ and the corresponding Jacobian have to be split, resulting in:

$$
\tau_{\text {ext }}=\sum_{i=1}^{k}\left[\begin{array}{c}
\tau_{l_{i}} \\
\tau_{u_{i}}
\end{array}\right]=\sum_{i=1}^{k}\left[\begin{array}{c}
\mathbf{J}_{l}^{\top}\left(\mathbf{x}_{\mathbf{b}}\right) \mathbf{W}_{N P_{i}} \\
\mathbf{J}_{u_{i}}^{\top}(\theta) \mathbf{F}_{P_{i}}
\end{array}\right]
$$

where $\mathbf{F}_{P_{i}} \in \mathbb{R}^{3}$ is the "properly propagated" component of $\mathbf{F}_{\text {ext }}$, generating satisfactory torques along the considered limb's kinematic chain without violating any physical constraint of the robot, $\mathbf{W}_{N P_{i}}=\left[\mathbf{F}_{N P_{i}}, \mathbf{M}_{N P_{i}}\right]^{\top}=$ $\left[\mathbf{F}_{N P_{i}}, \mathbf{F}_{N P_{i}} \times \mathbf{t}_{i}\right]^{\top} \in \mathbb{R}^{6}$ is the wrench generated by "weakly propagated" components $\mathbf{F}_{N P_{i}} \in \mathbb{R}^{3}$ of $\mathbf{F}_{\text {ext }}$ and $\mathbf{t}_{i}$ is the vector of application point in the arm base frame. $\mathbf{J}_{l}\left(\mathbf{x}_{\mathbf{b}}\right) \in \mathbb{R}^{6 \times 3}$ and $\mathbf{J}_{u_{i}}(\theta) \in \mathbb{R}^{3 \times n}$ are respectively the lower and upper limb Jacobian matrices. Unlike the $\mathbf{J}_{u_{i}}(\theta)$, varying for each contact point on the upper limbs, $\mathbf{J}_{l}\left(\mathbf{x}_{\mathbf{b}}\right)$ is unique since the contact point between upper and lower limbs remain the same. The main challenge in this work is therefore to correctly choose the components $\mathbf{F}_{P_{i}}$ and $\mathbf{F}_{N P_{i}}$ of $\mathbf{F}_{e x t_{i}}$ in order to achieve human-like whole-body compliance. 


\section{Constrained Force Propagation}

\section{A. Nullspace forces}

When an external force is applied to one of the robot's upper limbs, it propagates along the corresponding kinematic chain as a set of reactive torques $\tau_{u_{i}}=\mathbf{J}_{u_{i}}^{\top}(\theta) \mathbf{F}_{e x t_{i}}$. In order to make a given robot whole-body compliant - without any care of its kinematic or dynamic constraints - the nullspace components $\mathbf{F}_{\text {null }_{i}}$ of external forces $\mathbf{F}_{\text {ext }}$, resulting in $\tau_{u_{i}}=\mathbf{J}_{u_{i}}^{\top}(\theta) \mathbf{F}_{\text {null }_{i}}=\mathbf{0}_{n \times 1}$, must be directly projected onto the mobile base in order to generate suitable reactive motions: $\mathbf{F}_{N P_{i}}=\mathbf{F}_{\text {null }_{i}}$. In practice, the simplest way to determine these nullspace components is to compute their orthogonal complementary: $\mathbf{F}_{\text {null }_{i}}^{\perp}=\mathbf{F}_{\text {ext }_{i}}-\mathbf{F}_{\text {null }_{i}}$. As shown in Fig. 3.a) and 3.b), finding $\mathbf{F}_{\text {null }_{i}}^{\perp}$ can be understood as finding the minimum L2-norm force producing the torque $\tau_{u_{i}}=\mathbf{J}_{u_{i}}^{\top}(\theta) \mathbf{F}_{\text {ext }}=\mathbf{J}_{u_{i}}^{\top}(\theta) \mathbf{F}_{\text {null }_{i}}^{\perp}$ along the upperlimbs. This can be conveniently formulated using Quadratic Programming (QP):

$$
\begin{aligned}
\mathbf{F}_{\text {null }_{i}}^{\perp}=\min _{\mathbf{F}_{Q P}} & \left\|\mathbf{F}_{Q P}\right\|_{2}^{2} \\
\text { s.t. } & \mathbf{J}_{u_{i}}^{\top} \mathbf{F}_{Q P}=\tau_{u_{i}}
\end{aligned}
$$

where $\mathbf{F}_{Q P} \in \mathbb{R}^{m}$ is the optimization variable. The main issue here, is that the problem is often overdetermined since the number of columns of $\mathbf{J}_{u_{i}}$ can be greater than the dimension of the applied force vector (depending on the external force application point in the robot arm kinematic chain). The resulting QP has therefore either a unique solution (namely $\mathbf{F}_{\text {null }_{i}}^{\perp}=\mathbf{F}_{\text {ext }_{i}}$ ) or no solution at all. In these conditions, taking the robot's kinematic and dynamic constraints into account is difficult since the QP fails to find a solution whenever a single constraint is violated. It is nevertheless possible to fix this issue by defining a slack variable $\delta \tau \in \mathbb{R}^{n}$, $\delta \tau<<\mathbf{1}_{n \times 1}$ such as:

$$
\begin{aligned}
\mathbf{F}_{P_{i}}=\min _{\mathbf{F}_{Q P}} & \left\|\mathbf{F}_{Q P}\right\|_{2}^{2} \\
\text { s.t. } & \tau_{u_{i}}-\delta \tau \leq \mathbf{J}_{u_{i}}^{\top} \mathbf{F}_{Q P} \leq \tau_{u_{i}}+\delta \tau
\end{aligned}
$$

Since in this formulation the QP can have many solutions, it is therefore more robust to kinematic or dynamic constraints. However, the greater the value of $\delta \tau$, the lower the precision: $\delta \tau$ must therefore be properly tuned.

\section{B. Forces applied on close-to-singular configurations}

As explained above, if an external force is applied along a singular direction of a robot manipulator, the corresponding torque on its kinematic chain will be zero. The minimal L2norm force generating this torque will therefore also be zero, which implies - according to the eq.(4) - that every external force component applied along this singular direction will be directly projected onto the mobile base. Unfortunately, the problem is more complex in the vicinity of a singularity since the force required to generate movement along the so called "singular direction" increases dramatically, as it becomes more and more difficult to generate suitable torque
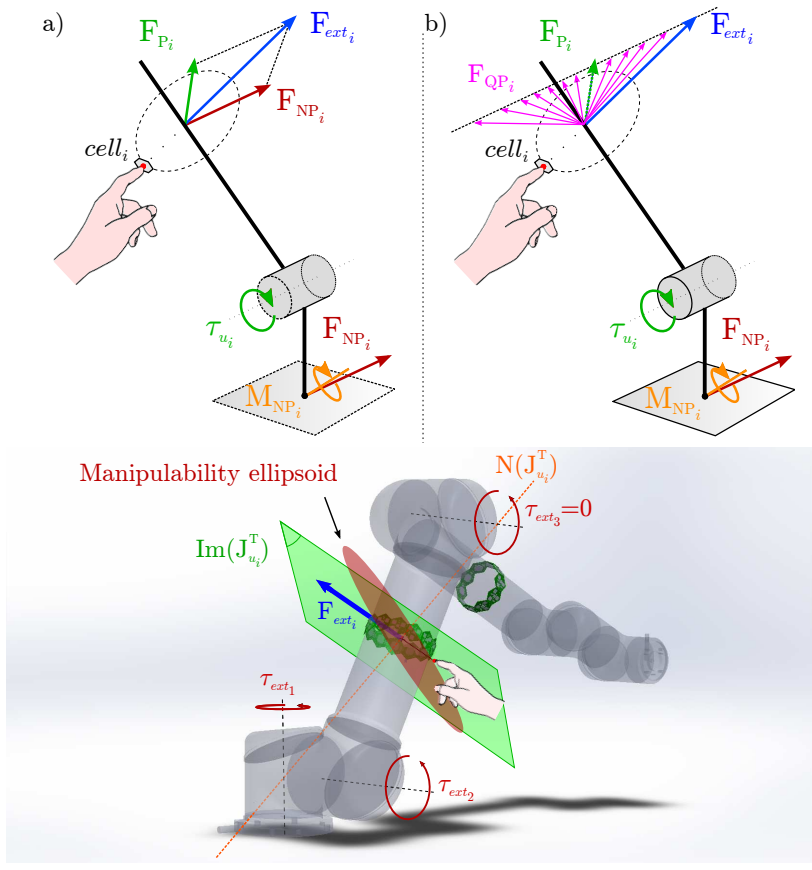

Fig. 3. a): Simplified example of propagation of the nullspace component of applied force onto the mobile base. b): Finding the rangespace component $\mathbf{F}_{\mathbf{W}_{i}}$ of $\mathbf{F}_{i}$ can be formulated as an optimization problem, namely find the smallest force $\mathbf{F}_{Q P}$ which, applied on the same point of the robot structure, generates the torque $\tau_{i}$ produced by $\mathbf{F}_{i} . \mathrm{c}$ ): Nullspace and Rangespace of a UR5 manipulator computed with the QP in the vicinity of a singularity (on the second joint). The corresponding manipulability ellipsoid (in red on the figure) is strongly oblong due to the proximity of a singularity. It is therefore hard to move the manipulator along the corresponding direction of task space (even if this direction does not belong to the nullspace of the Jacobian transpose). The external force components applied along this direction must therefore be treated as nullspace forces and projected onto the mobile base in order to guarantee safe interaction.

on the corresponding robot joints (Fig. 3.c). For obvious security reasons, these so called weakly propagated force components must also be directly projected onto the mobile base, along with the forces applied on singular configurations. Identifications of weakly propagated external forces can be achieved by computing the manipulability or force ellipsoid [17] of the Jacobian matrix associated with the force contact point. When one of the main axes of the manipulability (respectively force) ellipsoid is becoming "small" (respectively "large") - i.e. when the associated singular value $\sigma_{i}$ of the Jacobian matrix obtained by Singular Value Decomposition (SVD) lies under a predefined threshold - they are considered as being part of $\mathbf{F}_{N P_{i}}$. The orthogonal projections of external forces along these axis must therefore be directly propagated onto the mobile base. If this method is very intuitive, it is however difficult to apply in practice due to computational cost considerations since one SVD has to be computed for each active skin cell. In our case, the ratio $\eta$ between $L_{2}$ norm of the measured force vector and $L_{2}$-norm of the corresponding torque, gives promising results while requiring a lower computational cost:

$$
\eta=\frac{\|\mathbf{F}\|_{2}}{\|\tau\|_{2}+\varepsilon} \in \mathbb{R}_{+}
$$


Here $\varepsilon<<1$ aims to avoid ill conditioning, occurring when a force is applied onto a pure singular limb configuration. By reshaping this criterion with a sigmoid function, it is possible to reformulate the eq. (5) as:

$$
\begin{aligned}
\underset{\mathbf{F}_{Q P}}{\min } & \left\|\mathbf{F}_{Q P}\right\|_{2}^{2} \\
\text { s.t. } & (1-\alpha) \tau_{u_{i}}-\delta \tau \leq \mathbf{J}_{u_{i}}^{\top} \mathbf{F}_{Q P} \leq(1-\alpha) \tau_{u_{i}}+\delta \tau
\end{aligned}
$$

where $\forall \nu, \eta_{0} \in \mathbb{R}_{+}^{*}$ :

$$
\left.\alpha=\frac{1}{1+e^{-\nu\left(\eta-\eta_{0}\right)}} \in\right] 0 ; 1[
$$

In this way, when a force is applied on a close-to-singular configuration, i.e. when $\eta>\eta_{0}$, we have $\alpha \rightarrow 1$ and therefore $(1-\alpha) \tau_{u_{i}} \pm \delta \tau \rightarrow \pm \delta \tau$. The optimization problem is therefore equivalent to:

$$
\begin{array}{cl}
\min _{Q P} & \left\|\mathbf{F}_{Q P}\right\|_{2}^{2} \\
\text { s.t. } & -\delta \tau \leq \mathbf{J}_{u_{i}}^{\top} \mathbf{F}_{Q P} \leq \delta \tau
\end{array}
$$

with the trivial solution $\mathbf{F}_{P_{i}}=\mathbf{0}_{m \times 1}$ and therefore $\mathbf{F}_{N P_{i}}=$ $\mathbf{F}_{\text {ext }}$. The external force is therefore entirely propagated onto the mobile base. Similarly, when the applied force is properly propagated - i.e. not applied in the vicinity of a singular configuration - we have $\eta<\eta_{0}$ and thus $\alpha \rightarrow 0$, resulting in $(1-\alpha) \tau_{u_{i}} \pm \delta \tau \rightarrow \tau_{u_{i}} \pm \delta \tau$. The optimization problem is therefore now equivalent to the formulation of eq.(5).

\section{Robot physical limitations}

As every mechanical systems, robots are submitted to a set of kinematic and dynamic constraints, depending - in particular - on their geometry or actuation strategy. When a robot reaches one of its mechanical limits, the torque applied onto the considered joints is automatically set to zero by internal safety checks, resulting in a temporary loss one or several DOF. Nevertheless, maintaining compliance in this context is still possible, provided that the robot's constraints are properly taken into account in the main control loop. In this way, the forces applied in the vicinity of a mechanical limit can be identified and directly projected onto the mobile base, thereby generating suitable compliant reactive motions. Since most robot kinematic and dynamic constraints can formulated as a set of inequalities, they can therefore be naturally taken into account in the previous QP formulation. As proposed in [18], we here consider a set of joint space constraints, namely torque limits, joint limits and velocity limits:

$$
\begin{aligned}
-\tau_{\max } & \leq \mathbf{J}_{u_{i}}^{\top} \mathbf{F}_{Q P} \leq \tau_{\max } \\
\lambda\left(\theta_{\lim L}-\theta\right) & \leq \mathbf{J}_{u_{i}}^{\top} \mathbf{F}_{Q P} \leq \lambda\left(\theta_{\lim U}-\theta\right) \\
\mu\left(\dot{\theta}_{\lim L}-\dot{\theta}\right) & \leq \mathbf{J}_{u_{i}}^{\top} \mathbf{F}_{Q P} \leq \mu\left(\dot{\theta}_{\lim U}-\dot{\theta}\right)
\end{aligned}
$$

where $\lambda, \mu \in \mathbb{R}$ are convergence rates and where $\theta_{\text {lim } L}$, $\theta_{\text {limU }} \dot{\theta}_{\text {lim } L}$ and $\dot{\theta}_{\text {limU }} \in \mathbb{R}^{n}$ are lower and upper joint position and velocity limits vectors for the robot's upper kinematic chains. Task space constraints such as self-collision avoidance can also be formulated in a similar way, by directly bounding $\mathbf{F}_{Q P}$. The $\mathrm{QP}$ for the constrained force


propagation problem can eventually be formulated as:

$$
\begin{array}{cl}
\min _{\mathbf{F}_{Q P}} & \left\|\mathbf{F}_{Q P}\right\|_{2}^{2} \\
\text { s.t. } & (1-\alpha) \tau_{u_{i}}-\delta \tau \leq \mathbf{J}_{u_{i}}^{\top} \mathbf{F}_{Q P} \leq(1-\alpha) \tau_{u_{i}}+\delta \tau \\
& \lambda\left(\theta_{\text {limL }}-\theta\right) \leq \mathbf{J}_{u_{i}}^{\top} \mathbf{F}_{Q P} \leq \lambda\left(\theta_{\text {limU }}-\theta\right) \\
& \mu\left(\dot{\theta}_{\text {lim } L}-\dot{\theta}\right) \leq \mathbf{J}_{u_{i}}^{\top} \mathbf{F}_{Q P} \leq \mu\left(\dot{\theta}_{\text {lim }}-\dot{\theta}\right) \\
& -\tau_{\max } \leq \mathbf{J}_{u_{i}}^{\top} \mathbf{F}_{Q P} \leq \tau_{\max }
\end{array}
$$

When one of the upper limbs gets close to a mechanical limit, the torque generated on the involved joints by the external forces is automatically bounded. The QP-solver then tries to find the minimal force satisfying these constraints and propagates its complementary onto the mobile base. If the solver fails to find such a solution, the applied force will be entirely propagated onto the mobile base. The resulting framework is therefore both versatile and easy to use, the only challenge being to correctly formulate the constraints. 

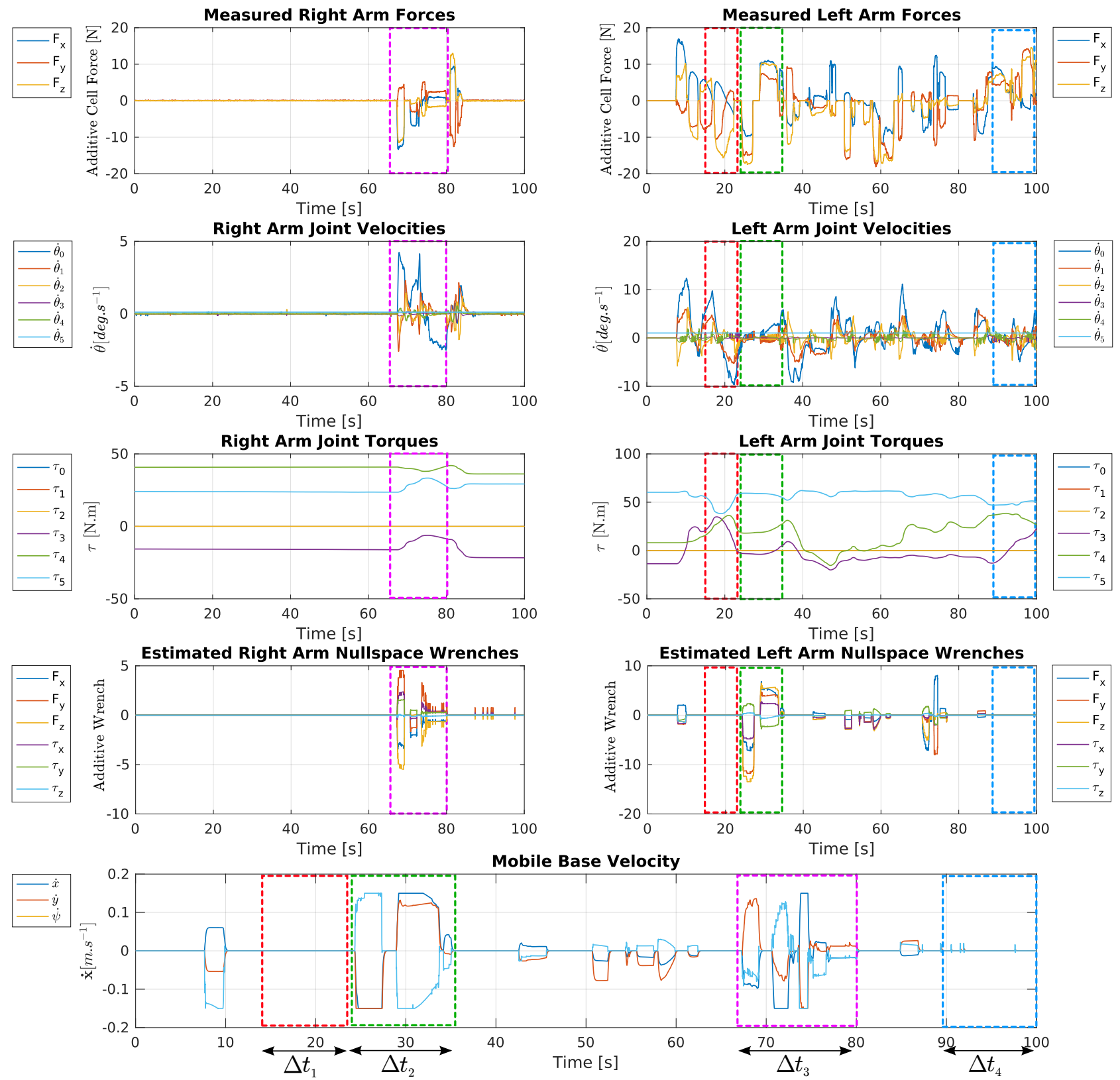

Fig. 4. Experiment Results. The graphs of first row shows the summed contributions of skin cells forces in [N] for both arms. The forces measured by skin cells are originally on a 0 to 1 scale (without unit). The final force is computed as: $\mathbf{F}=K_{f} \cdot \mathbf{F}_{\text {cell }}+K_{d} \cdot \mathbf{d}_{c e l l}$ where $K_{f} \equiv[N]$ and $K_{d} \equiv[N . m]$. The graphs in second and third rows show respectively the resulting joint velocities in [deg.s $\left.{ }^{-1}\right]$ and torques in $[N . m]$ for both arms. The estimated improperly propagated wrench components are shown in the fourth row. Finally, the corresponding mobile base velocities in $\left[m . s^{-1}\right]$, obtained after propagation of these wrench components are shown in the last row graph. For each plot, the $\mathrm{x}$-axis represents time in seconds.

\section{EXPERIMENTS}

\section{A. Implementation Details and Hardware}

The robot platform TOMM (Fig. 1 and Fig. 2.a) was developed at our institute: it is an assembly of two "UR5" 6-DOF industrial robots (from Universal Robots), covered with 600 multimodal artificial skin cells and mounted on a holonomic mobile base. The artificial skin is organized in patches, one patch covering each limb segment. Since the relative positions of skin cells are known in a given patch (using network exploration), it is possible after a short calibration step to compute each cell's homogeneous transforms with respect to the patch frame. As a result, the 3D shape (Fig. 2.d) of a given patch can be reconstructed and each cell can be associated with a specific Jacobian matrix $\mathbf{J}_{\text {cell }_{i}}$ when the patch is mounted on a robot [19]. Therefore, not only the force but also its application point can be known in the same time. For each control update it is necessary to browse active cells in each patch and to sum-up their corresponding force contributions as exposed in the algorithms 1 and 2. While the amount of generated data is substantial, it is nevertheless handled very efficiently [20], thereby guaranteeing extremely low latency. TOMM's central unit is based on two intel 
$i 7$ computers with $16 \mathrm{~Gb}$ of RAM each: the first unit is dedicated to control tasks while the second one is used for machine learning and vision. TOMM's arms as well as its mobile base are velocity-controlled. However, force/torque based control can still be realized using an admittance control layer in order to convert the desired torque commands into suitable velocity commands. The force/torque control loop is running at $125 \mathrm{~Hz}$. We used the $\mathrm{C}++$ library qPOASES [21] as QP-solver since it provides interesting timing features for implementation in real-time control loops. Since one QP has to be solved for each activated skin cell, the computational cost increases linearly with the number of active cells. In addition to the hierarchical compliance controller, we defined a gravity compensation task for both arms: $\tau_{c t r l}=\mathbf{G}(\mathbf{q})$. During the experiments, the parameters $\eta_{0}, \nu$ and $\delta \tau$ of eq. (7) and eq. (5) were respectively set to $\eta_{0}=7, \nu=3$ and $\delta \tau=0.01 \cdot \mathbf{1}_{n \times 1}$.

\section{B. Experiment description and Results}

During this experiment a set of forces is applied on TOMM's arms by an external operator, generating reactive compliant motions on the robot. The Figure 4 shows the experiment results. It is possible to notice for $\Delta t_{1}=[\mathbf{1 4} ; \mathbf{s} ; \mathbf{2 4}]$ that an external force is applied onto the left robot arm, thereby triggering compliant motion. This force is maintained until the arm reaches a close-to-singular configuration. Another set of forces is then applied close to the newly created "singular axis" for $\Delta t_{2}=[\mathbf{2 4 \mathbf { s }} ; \mathbf{3 6 \mathbf { s }}]$. Since these forces cannot be properly propagated using only the arms, they are projected onto the mobile base, in order to generate suitable compliant motions. At $\Delta t_{3}=[\mathbf{6 8} \mathbf{s} ; 80 \mathbf{s}]$, a set of external forces is applied close to the root of the right arm's kinematic chain. Since most of these force are poorly propagated on the arm's kinematic chain - according to the criterion of eq.(6) - they are then to be projected onto the mobile base in order to generate compliant reactive motions. Finally for $\Delta t_{4}=[\mathbf{9 0 s} ; \mathbf{1 0 0 s}]$, a set of external forces is applied to the left arm, far from any singular direction or physical limit. We can observe that since proper reactive torques are generated onto the arm joints, the mobile base does not moves. The hierarchical force propagation algorithm therefore seems to behave in accordance with our expectations. A video demonstrating the robustness of the controller is provided as a complement to this paper. ${ }^{1}$

\section{CONCLUSION}

In this paper, we address the issue of tactile-based hierarchical compliance with constrained force propagation for mobile manipulators. Using quadratic programing, we propose a method to estimate the ill-propagated force components on the arm kinematic chains. These components are then projected onto the mobile base in order to generate safe reactive motions. The experiments performed on a dual arm mobile manipulator eventually demonstrated the reliability and the robustness of this method.

\section{REFERENCES}

[1] N. Hogan, "Impedance control: An approach to manipulation," in American Control Conference, 1984, pp. 304-313, IEEE, 1984.

[2] O. Khatib, "A unified approach for motion and force control of robot manipulators: The operational space formulation," Robotics and Automation, IEEE Journal of, vol. 3, no. 1, pp. 43-53, 1987.

[3] C. Loughlin, A. Albu-Schäffer, S. Haddadin, C. Ott, A. Stemmer, T. Wimböck, and G. Hirzinger, "The dlr lightweight robot: design and control concepts for robots in human environments," Industrial Robot: an international journal, vol. 34, no. 5, pp. 376-385, 2007.

[4] A. Albu-Schäffer, O. Eiberger, M. Fuchs, M. Grebenstein, S. Haddadin, C. Ott, A. Stemmer, T. Wimböck, S. Wolf, C. Borst, et al., "Anthropomorphic soft robotics-from torque control to variable intrinsic compliance," in Robotics research, pp. 185-207, Springer, 2011.

[5] T. L. Chen and C. C. Kemp, "A direct physical interface for navigation and positioning of a robotic nursing assistant," Advanced Robotics, vol. 25, no. 5, pp. 605-627, 2011.

[6] T. L. Chen, T. Bhattacharjee, J. L. McKay, J. E. Borinski, M. E. Hackney, L. H. Ting, and C. C. Kemp, "Evaluation by expert dancers of a robot that performs partnered stepping via haptic interaction," PloS one, vol. 10, no. 5, p. e0125179, 2015.

[7] A. Dietrich, T. Wimböck, A. Albu-Schäffer, and G. Hirzinger, "Reactive whole-body control for dynamic mobile manipulation,"

[8] A. Dietrich, K. Bussmann, F. Petit, P. Kotyczka, C. Ott, B. Lohmann, and A. Albu-Schäffer, "Whole-body impedance control of wheeled mobile manipulators," Autonomous Robots, pp. 1-13, 2015.

[9] A. Dietrich, T. Wimbock, A. Albu-Schaffer, and G. Hirzinger, "Integration of reactive, torque-based self-collision avoidance into a task hierarchy," IEEE Transactions on Robotics, vol. 28, no. 6, pp. 1278 1293, 2012.

[10] L. Saab, Generating whole body movements for dynamic anthropomorphic systems under constraints. PhD thesis, Université Paul SabatierToulouse III, 2011.

[11] K. S. Kim, A. S. Kwok, G. C. Thomas, and L. Sentis, "Fully omnidirectional compliance in mobile robots via drive-torque sensor feedback," in 2014 IEEE/RSJ International Conference on Intelligent Robots and Systems, pp. 4757-4763, IEEE, 2014.

[12] J. Frémy, F. Ferland, M. Lauria, and F. Michaud, "Force-guidance of a compliant omnidirectional non-holonomic platform," Robotics and Autonomous Systems, vol. 62, no. 4, pp. 579-590, 2014.

[13] F. Ferland, D. Létourneau, A. Aumont, J. Frémy, M.-A. Legault, M. Lauria, and F. Michaud, "Natural interaction design of a humanoid robot," Journal of Human-Robot Interaction, vol. 1, no. 2, pp. 118 134, 2012.

[14] G. Cannata, M. Maggiali, G. Metta, and G. Sandini, "An embedded artificial skin for humanoid robots," in Multisensor Fusion and Integration for Intelligent Systems, 2008. MFI 2008. IEEE International Conference on, pp. 434-438, IEEE, 2008.

[15] P. Mittendorfer and G. Cheng, "Humanoid multimodal tactile-sensing modules," Robotics, IEEE Transactions on, vol. 27, no. 3, pp. 401-410, 2011.

[16] M. W. Spong and M. Vidyasagar, Robot dynamics and control. John Wiley \& Sons, 2008.

[17] T. Yoshikawa, "Dynamic manipulability of robot manipulators," in Robotics and Automation. Proceedings. 1985 IEEE International Conference on, vol. 2, pp. 1033-1038, IEEE, 1985.

[18] E. Lutscher and G. Cheng, "Hierarchical inequality task specification for indirect force controlled robots using quadratic programming," in Intelligent Robots and Systems (IROS 2014), 2014 IEEE/RSJ International Conference on, pp. 4722-4727, IEEE, 2014.

[19] P. Mittendorfer and G. Cheng, "3d surface reconstruction for robotic body parts with artificial skins," in Intelligent Robots and Systems (IROS), 2012 IEEE/RSJ International Conference on, pp. 4505-4510, IEEE, 2012

[20] F. Bergner, P. Mittendorfer, E. Dean-Leon, and G. Cheng, "Event-based signaling for reducing required data rates and processing power in a large-scale artificial robotic skin," in Intelligent Robots and Systems (IROS), 2015 IEEE/RSJ International Conference on, pp. 2124-2129, IEEE, 2015

[21] H. J. F. et al., "qpoases user's manual - version 3.0." http://www.coinor.org/qpOASES/doc/3.0/manual.pdf, 2014. 